\title{
Acquiring palm vein patterns for visual interpretation
}

\begin{abstract}
This paper presents the setup required in acquiring palm vein pattern for visual interpretation based on the use of near infrared (NIR) spectrum illumination. It uses the NIR peak wavelengths of $0.830 \mathrm{\varepsilon m}, 0.850 \mathrm{\varepsilon m}, 0.870 \mathrm{\varepsilon m}, 0.880 \mathrm{\varepsilon m}$ and $0.890 \mathrm{\varepsilon m}$ in the experiment with variation to the number of layers used as the filtering material. The effect of the NIR peak wavelengths are observed and discussed, in terms of the visibility of the vein pattern in the image acquired. Other issues that influence the visibility of the vein pattern in the developed setup are also presented. The developed experimental setup can be extended its usage in acquiring vein pattern in other parts of human body, which can be used for biometric, medical or device prototyping purpose.
\end{abstract}

Keyword: Palm vein pattern; Image acquisition; Near infrared spectrum 